\title{
Global asymptotics of filtration in porous media
}

\author{
Liudmila Kuzmina ${ }^{1}$, Yuri Osipov ${ }^{2, *}$ and Yulia Zheglova ${ }^{2}$ \\ ${ }^{1}$ National Research University Higher School of Economics, Moscow, Russia \\ ${ }^{2}$ Moscow State University of Civil Engineering, Yaroslavskoe shosse, 26, Moscow, 129337, Russia
}

\begin{abstract}
Filtration problems are actual for the design of underground structures and foundations, strengthening of loose soil and construction of watertight walls in the porous rock. A liquid grout pumped under pressure penetrates deep into the porous rock. Solid particles of the suspension retained in the pores, strengthen the loose soil and create watertight partitions. The aim of the study is to construct an explicit analytical solution of the filtration problem. A one-dimensional model of deep bed filtration of a monodisperse suspension in a homogeneous porous medium with size-exclusion mechanism of particles retention is considered. Solid particles are freely transferred by the carrier fluid through large pores and get stuck in the throats of small pores. The mathematical model of deep bed filtration includes the mass balance equation for suspended and retained particles and the kinetic equation for the deposit growth. The model describes the movement of concentrations front of suspended and retained particles in an empty porous medium. Behind the concentrations front, solid particles are transported by a carrier fluid, accompanied by the formation of a deposit. The complex model has no explicit exact solution. To construct the asymptotic solution in explicit form, methods of nonlinear asymptotic analysis are used. The new coordinate transformation allows to obtain a parameter that is small at all points of the porous sample at any time. In this paper, a global asymptotic solution of the filtration problem is constructed using a new small parameter. Numerical calculations are performed for a nonlinear filtration coefficient found experimentally. Calculations confirm the closeness of the asymptotics to the solution in the entire filtration domain. For a nonlinear filtration coefficient, the asymptotics is closer to the numerical solution than the exact solution of the problem with a linear coefficient. The analytical solution obtained in the paper can be used to analyze solutions of problems of underground fluid mechanics and fine-tune laboratory experiments.
\end{abstract}

\section{Introduction}

The problems associated with the transport and deposition of solid microscopic particles in porous media are actual in nature, science and technology. Pollution and bioremediation of water reservoirs, purification of industrial effluents and liquid municipal waste, operation of air filters, a drop in oil production caused by reduction of rock permeability are associated

*Corresponding author: yuri-osipov@mail.ru 
with filtration of the suspension in porous media [1, 2]. In the construction industry, in order to create a waterproof layer, a solution of bentonite is poured into a porous soil, which is filtered in pores and, while hardening and expanding, creates a reliable barrier to formation and flood waters [3].

The paper considers a one-dimensional model of filtration of a monodisperse suspension in a homogeneous porous medium. Suspended solid particles are transported by the carrier fluid through the pores, with some particles blocking and forming a deposit. Depending on the structure and properties of the porous medium and suspended particles, diffusion, electrostatic, gravitational and hydrodynamic forces, etc., affect the particles retention. [4-6]. If the distributions of particle sizes and pores overlap, the size-exclusion mechanism of particle retention plays the main role: suspended particles freely pass through large pores and get stuck in small pores that are smaller than the particle diameter. It is assumed that the retained particles cannot be knocked out of the pore by other particles or by the flow of a carrier fluid [7].

The mathematical model of deep bed filtration includes the mass balance equation for suspended and retained particles and the kinetic equation for deposit growth [8]. The solution of a nonlinear hyperbolic system of the first order partial differential equations are the volume concentrations of suspended and retained particles. In some cases, it is possible to obtain analytical exact or asymptotic solutions of the filtration problem $[9,10]$.

Asymptotics is constructed as an expansion in a small parameter. In this problem, such a parameter may be the distance from porous medium inlet or from the concentration front, the reciprocal of a large time, etc. The constructed asymptotic solutions are local, i.e. applicable only in a specific domain. The crosslinking of local asymptotics into the global one is prevented by the presence of domains where no asymptotic solutions are applicable. In this paper a global asymptotics applicable in the entire filtration domain is obtained. A new small parameter of the asymptotic solution is a function of the coordinate. This parameter is small at all points of the porous medium for any time. Numerical calculations confirm the closeness of the asymptotics to the solution.

Section 2 is devoted to the governing equations of deep bed filtration. In Section 3, the global asymptotics is constructed. Results of numerical calculations are given in Section 4. Discussion and Conclusions in Sections 5 and 6 complete the paper.

\section{Governing equations}

In the domain

$$
\Omega=\{(x, t): 0 \leq x \leq 1, t \geq 0\}
$$

the concentrations $C(x, t)$ of suspended particles and $S(x, t)$ of retained particles satisfy the unidimensional system of equations

$$
\begin{aligned}
& \frac{\partial C}{\partial t}+\frac{\partial C}{\partial x}+\frac{\partial S}{\partial t}=0 ; \\
& \frac{\partial S}{\partial t}=\Lambda(S) C .
\end{aligned}
$$

The function $\Lambda(S)$, which determines the dependence of the particle retention rate on the amount of deposit, is called the filtration coefficient [11].

The boundary and initial conditions 


$$
\begin{gathered}
\left.C\right|_{x=0}=1 ; \\
\left.C\right|_{t=0}=0 ;\left.\quad S\right|_{t=0}=0
\end{gathered}
$$

determine the unique solution of the system (1), (2). Condition (3) corresponds to the injection of a suspension of constant concentration at the porous medium inlet. According to condition (4), at the initial time, the porous medium does not contain any suspended and retained particles.

The concentrations front $t=x$ moves in a porous medium from the inlet $x=0$ to the outlet $x=1$ with constant velocity $v=1$. Before the front in the domain

$$
\Omega_{0}=\{(x, t): 0 \leq x \leq 1,0 \leq t<x\}
$$

the solution is zero; after the front in the domain

$$
\Omega_{S}=\{(x, t): 0 \leq x \leq 1, t \geq x\}
$$

the solution is positive. At the concentration front $t=x$, the solution $C(x, t)$ has a gap; the solution $S(x, t)$ is continuous in the whole domain $\Omega$ and

$$
\left.S\right|_{t=x}=0
$$

The transition to the characteristic variable

$$
\tau=t-x, \quad y=x
$$

allows to convert a wedge-shaped domain $\Omega_{S}$ into a half-strip

$$
\Omega_{S}^{\tau}=\{(x, t): 0 \leq x \leq 1, \tau \geq 0\}
$$

and to simplify the equations:

$$
\begin{aligned}
& \frac{\partial C}{\partial y}+\frac{\partial S}{\partial \tau}=0 \\
& \frac{\partial S}{\partial \tau}=\Lambda(S) C .
\end{aligned}
$$

The solution of the Goursat problem (6), (7) with conditions (3), (5) coincides with the solution of problem (1) - (4) in $\Omega_{S}$.

For blocking linear filtration coefficient (Langmuir coefficient)

$$
\Lambda(S)=a-b S
$$

this problem in $\Omega_{S}^{\tau}$ allows an exact solution [12]

$$
C(y, \tau)=\frac{e^{b \tau}}{e^{b \tau}+e^{a y}-1} ; \quad S(y, \tau)=\frac{a}{b} \cdot \frac{e^{b \tau}-1}{e^{b \tau}+e^{a y}-1} .
$$

In the case of a nonlinear filtration coefficient, there are no general explicit formulas for the solution [13]. Local asymptotics for the system (6), (7) are constructed in [14-16]. 


\section{Global asymptotic solution}

Consider the problem (6), (7), (3), (5) with a nonlinear filtration coefficient

$$
\Lambda(S)=a-b S+\varepsilon \Delta(S), a>0, b>0,
$$

where $\varepsilon>0$ is a small positive parameter.

After the change of the variables

$$
z=1-e^{-a y} ; T=1-e^{-b \tau}
$$

in the domain

$$
\Omega_{z, T}=\left\{0 \leq z \leq e^{-a}, 0 \leq T<1\right\}
$$

the system takes the form

$$
\begin{gathered}
\mathrm{a}(1-z) \frac{\partial C}{\partial z}+(a-b S+\varepsilon \Delta(S)) C=0 ; \\
b(1-T) \frac{\partial S}{\partial T}=(a-b S+\varepsilon \Delta(S)) C ; \\
\left.C\right|_{z=0}=1 ; \\
\left.S\right|_{T=0}=0 .
\end{gathered}
$$

In $\Omega_{z, T}$ the variable $z$ satisfies the inequality

$$
0 \leq z \leq 1-e^{-a}<1
$$

so it can be used as a small parameter for constructing the global asymptotics in $\Omega_{z, T}$.

The asymptotic solution of the problem (12) - (15) is constructed as a series in two small parameters $z$ and $\varepsilon$ :

$$
\begin{aligned}
& C(\varepsilon, z, T)=1+\sum_{i=0}^{n} \sum_{j=1}^{m} c_{i, j}(T) \varepsilon^{i} z^{j} / j !=1+z\left(c_{0,1}+\varepsilon c_{1,1}+\varepsilon^{2} c_{2,1}+\ldots\right)+ \\
& +\frac{z^{2}}{2}\left(c_{0,2}+\varepsilon c_{1,2}+\varepsilon^{2} c_{2,2}+\ldots\right)+\ldots \\
& S(\varepsilon, z, T)=\sum_{i=0}^{n} \sum_{j=0}^{m} s_{i, j}(T) \varepsilon^{i} z^{j} / j !=\left(s_{0,0}+\varepsilon s_{1,0}+\varepsilon^{2} s_{2,0}+\ldots\right)+ \\
& +z\left(s_{0,1}+\varepsilon s_{1,1}+\varepsilon^{2} s_{2,1}+\ldots\right)+\frac{z^{2}}{2}\left(s_{0,2}+\varepsilon s_{1,2}+\varepsilon^{2} s_{2,2}+\ldots\right)+\ldots
\end{aligned}
$$

Here, the coefficients $c_{i, j}, s_{i, j}$ depend only on $T$.

Substituting the expansions (17), (18) into equations (12), (13), and equating the terms with the same powers of $\varepsilon, z$, yields a recurrent system of ordinary differential equations for the coefficients $s_{i, j}$ and algebraic equations for $c_{i, j}$. The first equations are: 


$$
\begin{aligned}
& \varepsilon^{0} z^{0}: \quad b(1-T) s_{0,0}^{\prime}=\left(a-b s_{0,0}\right) \cdot 1 ; \quad a c_{0,1}+\left(a-b s_{0,0}\right) \cdot 1=0 \\
& \varepsilon^{0} z^{1}: \quad b(1-T) s_{0,1}^{\prime}=-b s_{0,1}+\left(\mathrm{a}-b s_{0,0}\right) c_{0,1} ; \quad a c_{0,2}-a c_{0,1}-b s_{0,1}+\left(\mathrm{a}-b s_{0,0}\right) c_{0,1}=0 \\
& \varepsilon^{1} z^{0}: \quad b(1-T) s_{1,0}^{\prime}=\left(-b s_{1,0}+\Delta\left(s_{0,0}\right)\right) ; \quad a c_{1,1}+\left(-b s_{1,0}+\Delta\left(s_{0,0}\right)\right)=0 .
\end{aligned}
$$

The initial conditions for differential equations follow from condition (15):

$$
\left.s_{i j}\right|_{T=0}=0 .
$$

The coefficients $c_{i, j}, s_{i, j}$ are obtained by successive solving of the equations (19) with conditions (20)

$$
\begin{aligned}
& s_{0,0}=\frac{a}{b} T ; \quad c_{0,1}=T-1 ; \quad s_{0,1}=\frac{a}{b} T(T-1) ; \quad c_{0,2}=2 T(T-1) ; \\
& s_{1,0}=\frac{1-T}{b} \int_{0}^{T} \frac{\Delta(a t / b)}{(t-1)^{2}} d t ; \quad c_{1,1}=\frac{1-T}{a} \int_{0}^{T} \frac{\Delta(a t / b)}{(t-1)^{2}} d t-\frac{1}{a} \Delta(a T / b) .
\end{aligned}
$$

Next asymptotic coefficients are defined similarly.

The asymptotic solution of the problem (12) - (15) is obtained by substituting coefficients (21) into expansions (17), (18).

\section{Numerical calculation}

Experiments on deep bed filtration of a monodisperse suspension in a homogeneous porous medium made it possible to determine the filtration coefficient for suspended particles of radius $r=3.168 \mu \mathrm{m}[17]$

$$
\Lambda(S)=1.551-3.467 \cdot 10^{-3} S-1.16 \cdot 10^{-6} S^{2}-1.16 \cdot 10^{-7} S^{3} .
$$

The function (22) can be represented in the form (10), where

$$
a=0.51 ; \quad b=5.956 \cdot 10^{-3} ; \quad \varepsilon=0,1 ; \Delta(S)=2.29 \cdot 10^{-5} S^{2}+1.35 \cdot 10^{-6} S^{3} .
$$

The numerical calculation of the problem (12) - (15) with coefficients (23) is performed by the finite difference method [18] using explicit scheme with steps $h_{x}=h_{t}=0.01$.

Figures 1, 2 show the graphs of the first-order asymptotics in $\varepsilon$ (blue line), the exact solution (9) for the linear filtration coefficient (8) (black) and the numerical solution (red). The greatest discrepancy of the graphs is observed at the porous medium outlet (Fig. 1, 2). 


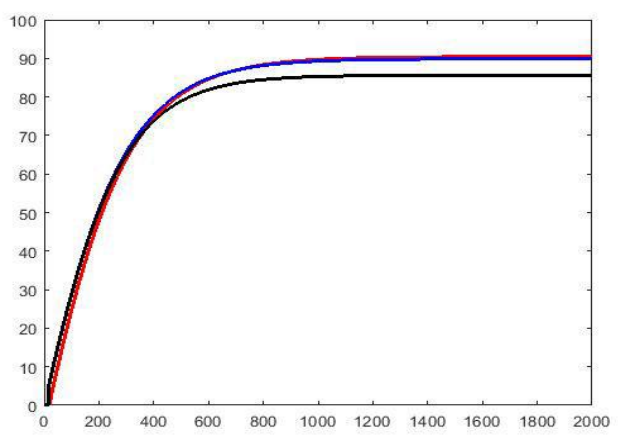

a)

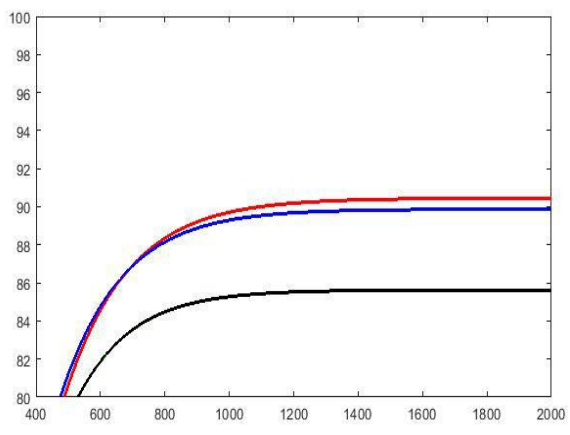

b)

Fig. 1. The retained concentration at the outlet $S(1, t)$ a) graphs completely; b) high resolution.

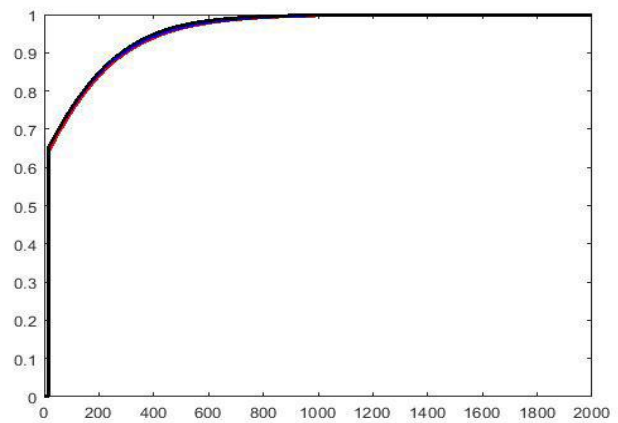

a)

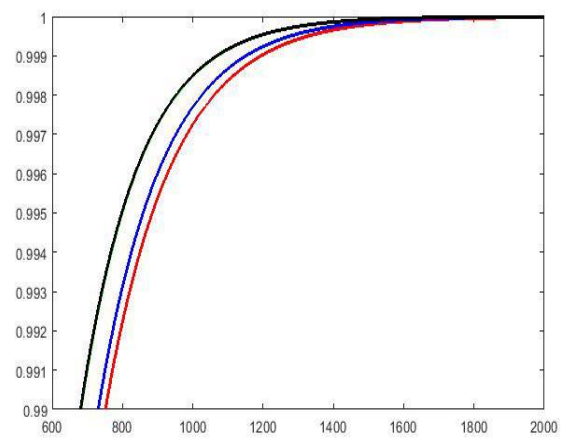

b)

Fig. 2. The suspended concentration at the outlet $C(1, t)$ a) graphs completely; b) high resolution.

For $t \leq 100$ the graphics are almost the same. Fig. 3 shows the graphs of the suspended and retained particles concentrations at time $t=1000$.

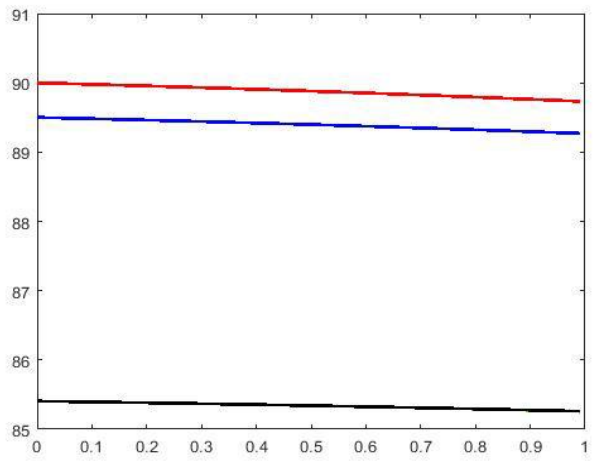

a)

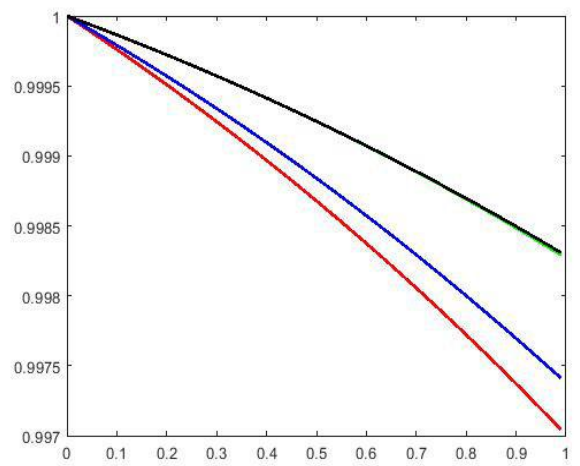

b)

Fig. 3. Particles concentrations at time $t=1000$ a) $S(x, 1000)$; b) $C(x, 1000)$.

The maximum errors of the asymptotics and the exact solution for the linear filtration coefficient are reached at the porous medium outlet $x=1$ : 


$$
\delta_{\text {asym }}^{S}=\frac{\left|S_{\text {num }}-S_{\text {asym }}\right|}{S_{\text {num }}}=0.0064 ; \quad \delta_{\text {lin }}^{S}=\frac{\left|S_{\text {num }}-S_{\text {lin }}\right|}{S_{\text {num }}}=0.0563 ; \quad \delta_{\text {asym }}^{C}=0.0015 ; \quad \delta_{\text {lin }}^{S}=0.0408
$$

\section{Discussion}

The model of deep bed filtration is determined by a nonlinear hyperbolic system of firstorder equations, which does not have an exact solution in explicit form. The global asymptotics allows to approximate the solution with explicit analytical formulas in the entire filtration domain. To reduce the error, the order of the asymptotic expansion should be increased.

More complex models assume the dependence of permissible porosity and fractional flow on the retained particles concentration [10]. The construction of the global asymptotic solution for this case is the subject of a separate study.

Filtration of the polydisperse suspension and chemical reactions of different particles with the formation of a deposit are described by similar equations [19]. Analytical solutions of such systems are known only for the simplest examples. This opens up wide possibilities for using nonlinear asymptotic methods.

\section{Conclusions}

A global asymptotic solution of the filtration problem for a nonlinear filtration coefficient is constructed. The asymptotics is close to the solution for all values of the coordinate $x$ and time $t$.

The expansion of the solution in a series using only one small parameter $\varepsilon$ with the nonlinear part of the filtration coefficient leads to a system of differential equations that do not have an explicit analytical solution. To obtain the asymptotics in explicit form, a new small parameter $z$ is used.

Explicit formulas for the asymptotic expansion in the small parameter $z=1-e^{-a x}$ are obtained.

For a nonlinear filtration coefficient, the global asymptotics is closer to the solution than the exact solution of the problem with a linear coefficient.

The small parameter $z$ of the asymptotic expansion increases with $x$ and reaches a maximum value $1-e^{-\Lambda(0)}$ at the porous medium outlet. At the outlet $x=1$, the asymptotic error is greatest.

These solutions make it possible to reduce the amount of experimental research and simplify the solution of the inverse problem - obtaining system coefficients from laboratory measurements of suspended particles concentrations at the porous medium outlet $[20,21]$.

\section{References}

1. D. C. Mays, J. R. Hunt, Environmental Science \&Technology, 39, 577-584 (2005)

2. P. Bedrikovetsky, Springer Science \& Business Media, 4, 600 (2013)

3. J. Yoon, C. S. El. Mohtar, Transport in Porous Media, 102(3), 365-385 (2014)

4. C. Tien, B. V. Ramarao, Granular filtration of aerosols and hydrosols (Amsterdam: 2nd ed. Elsevier, 2007)

5. C. V. Chrysikopoulos, V. I. Syngouna, Environmental Science \&Technology, 48, 6805-6813 (2014)

6. M. L. Martins-Costa, D. M. Alegre, F. B. F. Rachid, L. G. C. M. Jardim, RM. S. da Gama, International J. of Non-Linear Mechanics, 95, 168-177 (2017) 
7. J. P. Herzig, D. M. Leclerc, P. Legoff, Industrial and Engineering Chemistry, 62, 8-35 (1970)

8. Z. You, A. Badalyan, P. Bedrikovetsky, SPE J., 18, 620-633 (2013)

9. L. I. Kuzmina, Yu. V. Osipov, Y .P. Galaguz, International J. of Non-Linear Mechanics, 93, 1-6 (2017)

10. P. Bedrikovetsky, et al., Chemical Engineering J., 330, 1148-1159 (2017)

11. F. Civan, Reservoir formation damage (third ed. Gulf Professional Publishing, Burlington, MA, USA, 2014)

12. E. A. Vyazmina, P. G. Bedrikovetskii, A. D. Polyanin, Theoretical Foundations of Chemical Engineering, 41(5), 556-564 (2007)

13. Z. You, P. Bedrikovetsky, L. Kuzmina, Abstract and Applied Analysis, 680693 (2013)

14. Yu. Osipov, N. Kotov, IOP Conf. Series: Materials Science and Engineering, 365, 042006 (2018)

15. L. I. Kuzmina, Yu. V. Osipov, International J. for Computational Civil and Structural Engineering, 12(1), 158-163 (2016)

16. L. I. Kuzmina, Yu. V. Osipov, International J. for Computational Civil and Structural Engineering, 10(3), 17-22 (2014)

17. Z. You, et al., Chemical Engineering J., 258, 374-385 (2014)

18. Yu. Galaguz, G. Safina, IOP Conf. Series: Materials Science and Engineering, 365, 042005 (2018)

19. H. S. Fogler, Elements of chemical reaction engineering. 4th ed. (Prentice Hall: Upper Saddle River, NJ, 2006)

20. A. Vaz, P. Bedrikovetsky, P. D. Fernandes, A. Badalyan, T. Carageorgos, J. of Petroleum Science and Engineering, 151, 421-433 (2017)

21. A. C. Alvarez, G. Hime, J. D. Silva, D. Marchesin, Inverse Problems, 29, 025006 (2013) 